\title{
NOTE ON THE TRANSCENDENCE OF CERTAIN SERIES
}

WILLIAM J. LEVEQUE

1. Introduction. Let $k$ and $m$ be integers satisfying either of the sets of conditions, $k=0$ or $1, m \geqq 0$, or $k \neq 0,1$, and $m \geqq-1$. Then the series

$$
\sum_{n=0}^{\infty} \frac{(n+k)^{n+m} x^{n}}{n !}
$$

defines a function $f_{k m}$ in the range $|x|<e^{-1}$. It is the object of this note to show that $f_{k m}(x)$ is transcendental for every algebraic $x \neq 0$ in this interval. The proof involves no new transcendence investigations, but depends directly on Lindemann's theorem that $e^{x}$ is transcendental for algebraic $x \neq 0$. The connection between the exponential function and $f_{k m}$ is established by using the Lagrange expansion in powers of $x$

$$
G(z)=G(0)+\sum_{n=1}^{\infty} \frac{x^{n}}{n !}\left[D_{t}^{n-1} G^{\prime}(t) \phi^{n}(t)\right]_{t=0}
$$

of any function $G$ (regular in a neighborhood of $z=0$ ) of the quantity $z$ defined by the equation $x=z / \phi(z)$, where $\phi(z)$ is regular in a neighborhood of $z=0$ and $\phi(0) \neq 0$ (see Pólya-Szegö, Analysis, vol. 1, p. 125).

The symbol $0^{0}$ is to be interpreted as 1 , throughout. For brevity put $f_{k k}=f_{k}$.

\section{The case $k=0$.}

LEMMA 1. The function $y$ defined by the equation $y^{y}=e^{-x}$, and such that $\lim _{x \rightarrow 0} y(x)=y(0)=1$, is identical with $f_{-1}$ in the range $|x|<e^{-1}$.

For it follows from $y^{y}=e^{-x}$ that $x=z / e^{z}$, where $z=-\log y$. Taking $\phi(z)=e^{z}, G(z)=e^{-z}=y$ in (1) gives

$$
\begin{aligned}
y(x) & =1+\sum_{n=1}^{\infty} \frac{x^{n}}{n !}\left[D_{t}^{n-1}\left(-e^{(n-1) t}\right)\right]_{t=0} \\
& =1-\sum_{n=1}^{\infty} \frac{(n-1)^{n-1} x^{n}}{n !} \\
& =-f_{-1}(x) .
\end{aligned}
$$

Received by the editors May 24, 1950. 
LEMMA 2. $f_{0}(x)$ is transcendental for each algebraic $x$ with $0<|x|$ $<e^{-1}$.

By Lemma 1, $y=-f_{-1}$ is a solution of the equation $\log y=-x / y$, so that not both of $x, f_{-1}(x)$ can be algebraic unless $x=0$. But $y \log y$ $=-x$ implies that $y^{\prime}=y /(x-y)$, so that also not both of $y^{\prime}(x)$ $=-f_{0}(x)$ and $x \neq 0$ can be algebraic.

LEMMA 3. $f_{0 m}$ is a polynomial in $f_{0}$ with rational integral coefficients.

Let $y^{y}=e^{-x}$, and put $y^{\prime}=z=y /(x-y)$. Then it can be verified that $z^{\prime}=z^{2}(1+z) / x$, or

$$
f_{0}^{\prime}(x)=-\frac{f_{0}^{2}(x)\left(1-f_{0}(x)\right)}{x}
$$

so that $x D_{x} f_{0}=P_{1}\left(f_{0}\right)$, where $P_{1}$ is a polynomial with integral coefficients. It follows from this that $\left(x D_{x}\right)^{m} f_{0}=P_{m}\left(f_{0}\right)$, where $P_{m}$ is again a polynomial with integral coefficients. Finally, it is clear from the definition of $f_{k m}$ that $\left(x D_{x}\right)^{m} f_{0}=f_{0 m}$.

THEOREM 1. If $m \geqq 0, f_{0 m}(x)$ is transcendental for every algebraic $x$ for which $0<|x|<e^{-1}$.

This follows immediately from Lemmas 2 and 3.

It might mentioned that $f_{0}$ is a solution of the equation $(1-1 / y) e^{-(1-1 / y)}=x$.

3. The case $k \neq 0$. Putting $y=z, G(y)=e^{k y}, \phi(y)=e^{y}$ in (1) gives the following statement.

LEMMA 4. If $y$ is that solution of the equation $y e^{-y}=x$ which is continuous at $x=0$, then for $k \neq 0, e^{k y}=k f_{k,-1}(x)$ for $|x|<e^{-1}$.

Lemma 5. For $k \neq 1, f_{k 0}(x)$ is transcendental for each algebraic $x$ for which $0<|x|<e^{-1}$.

Since $f_{k-1,-1}^{\prime}=f_{k 0}$, it follows from Lemma 4 that

$$
e^{(k-1) y} y^{\prime}=f_{k 0},
$$

and since $y^{\prime}=e^{y} /(1-y)$,

$$
f_{k 0}(x)=\frac{e^{k y}}{1-y}=\frac{(y / x)^{k}}{1-y} .
$$

By its definition $y(x)$ is transcendental for algebraic $x \neq 0$, so that also $f_{k 0}(x)$ must be transcendental. 
Lemma 6. If $m \geqq 0, k \neq 0,1$, then

$$
f_{k m}(x)=\frac{P_{k m}(y)}{x^{k}}
$$

where $P_{k m}$ is a nonconstant rational function with integral coefficients and $y$ is the $y$ of Lemma 4 .

From the definition of $f_{k m}$, it is seen that

$$
\left(x D_{x}\right)^{m}\left(x^{k} f_{k 0}\right)=x^{k} f_{k m} .
$$

On the other hand, using the conventional operator notation, we have

$$
D_{x}=D_{x} y \cdot D_{y}=\frac{e^{y}}{1-y} D_{y}
$$

so that

$$
x D_{x}=\frac{y}{1-y} D_{y}
$$

Hence

$$
f_{k m}=\frac{1}{x^{k}}\left(\frac{y}{1-y} D_{y}\right)^{m} \frac{y^{k}}{1-y} ;
$$

the expression on the right contains a factor $y^{k}$ in the numerator, while the denominator is of the form $x^{k}(1-y)^{\imath}$, and the proof is complete.

LEMMA 7. For every $m$,

$$
x f_{1 m}(x)=f_{0 m}(x)-\left\{\begin{array}{lll}
0 & \text { if } & m \neq 0 \\
1 & \text { if } & m=0
\end{array}\right.
$$

and so for $m \geqq 0$, by Theorem $1, f_{1 m}(x)$ is transcendental for each algebraic $x, 0<|x|<e^{-1}$.

This follows immediately from the definition of $f_{k m}$.

THEOREM 2. If $k \neq 0,1$ and $m \geqq-1$, or if $k=1$ and $m \geqq 0$, the quantity $f_{k m}(x)$ is transcendental for each algebraic $x$ with $0<|x|<e^{-1}$.

UNIVERSITY OF MICHIGAN 